\title{
Response to influenza immunisation during treatment for cancer
}

\author{
J C Chisholm, T Devine, A Charlett, C R Pinkerton, M Zambon
}

\begin{abstract}
Aims-To assess the annual risk of influenza infection in children with cancer and the immunogenicity of a trivalent split virus influenza vaccine in these children. Methods-Eighty four children with cancer were tested for susceptibility to the circulating strains of influenza virus in autumn 1995 and 1996. Non-immunised children were reassessed the following spring for serological evidence of natural infection. Forty two patients received two doses of influenza vaccine. These children were receiving continuing chemotherapy for acute lymphoblastic leukaemia or were within six months of completing chemotherapy.

Results-Among the 84 children tested for influenza virus susceptibility only $8 \%$ of patients were fully protected (antibody titres $\geqslant 40$ ) against all three of the prevalent influenza virus strains; $33 \%$ were susceptible to all three viruses. Evidence of acquired natural infection was seen in $30 \%$ of unimmunised patients. Among immunised susceptible patients, $66 \%$ made some protective response to the vaccine and $55 \%$ showed protective antibody titres to all three viral strains following vaccination. Older age was associated with increased response to the $\mathrm{H} 1 \mathrm{~N} 1$ and H3N2 vaccine components, but total white cell count or neutrophil count at immunisation, type of cancer, or length of time on treatment for acute lymphoblastic leukaemia did not affect response.
\end{abstract}

Conclusions-Most children with cancer studied were at risk of influenza infection. A significant response to immunisation was seen, supporting annual influenza vaccination for children being treated for cancer.

(Arch Dis Child 2001;84:496-500)

Keywords: influenza; immunisation; susceptibility; cancer

Influenza is a common cause of respiratory tract infection and hospitalisation during the influenza season in adult patients with leukaemia, ${ }^{12}$ and following bone marrow transplantation, ${ }^{34}$ where a high incidence of complications, especially pneumonia, and significant mortality have been reported. Annual influenza infection rates in children vary from $18 \%$ to $48 \%,{ }^{5}$ but the infection is more common in children with cancer than in healthy control children. ${ }^{6}$ Although in children with cancer the illness usually runs a mild course it may result in hospitalisation and the interruption of cancer therapy. ${ }^{67}$ Severe and fatal complications have also been reported in these children. ${ }^{6-8}$

British $^{9}$ and American ${ }^{10}$ guidelines recommend annual influenza vaccination of high risk groups, including immunosuppressed children and adults, but uptake in high risk patients remains low. ${ }^{511}$ Contributing to the poor uptake is uncertainty over the efficacy of the vaccine in certain patient groups. ${ }^{5}$ Indeed, previous data suggest an impaired response to influenza immunisation in children on treatment for cancer compared with healthy children. ${ }^{12}$

This study is the first to report on the response to a trivalent split virion influenza vaccine in children during cancer therapy. The inclusion of a control, unvaccinated group in whom paired sera were obtained allowed assessment of the annual risk of disease in our population.

\section{Methods}

The study was undertaken over two successive influenza seasons, 1995-96 and 1996-97, at the Royal Marsden Hospital. Ethical approval for the study was obtained from the hospital ethics committee and informed, written consent was obtained from each child's parent.

In the first year an unselected group of all consenting patients attending the paediatric oncology outpatient department or resident on the paediatric inpatient ward between 28 September 1995 and 20 October 1995 was tested for susceptibility to the prevalent strains of influenza virus (A/Taiwan/1/86 [H1N1], A/Johannesburg/34/94 [H3N2], and B/Beijing/ $184 / 93$ [B]). Susceptibility was defined as antibody titre $<40$ (see below). Follow up serum was taken in May 1996 to document seroconversion in unimmunised patients. No attempt was made to document respiratory infections in these patients.

Among patients showing susceptibility to one or more of the prevalent virus strains, the following subgroups were offered immunisation in November 1995: (1) patients with acute lymphoblastic leukaemia (ALL) between weeks 9 and 18 of the UKALL XI treatment schedule (that is, on maintenance chemotherapy, between first and second intensification blocks); (2) patients with ALL between weeks 24 and 100 of the UKALL XI treatment schedule except those receiving the third intensification block (that is, patients on continuing maintenance chemotherapy); and (3) all patients who had finished chemotherapy within the past six months (including patients with leukaemia, solid tumours, and bone marrow transplant recipients). The following 
children were ineligible for vaccination: age less than 6 months; neutropenic (less than $1.0 \times$ $10^{9} / 1$ ) or lymphopenic (less than $1.0 \times 10^{9} / 1$ ) at time of vaccination; previously vaccinated against influenza; or egg allergy. Serum was taken to assess response four to six weeks after the second vaccination.

In the second study year vaccination was offered to the same subgroups of patients as above. The vaccine was also given to one patient with relapsed ALL and one child receiving chemotherapy for a brain tumour. Previously vaccinated patients were excluded. Prevaccination and 4-6 week post-vaccination sera were obtained, although, in this cohort, the vaccine was given without knowing the immune status.

All immunised children received two doses of inactivated influenza vaccine (split virion; Aventis Pasteur MSD), subcutaneously, four weeks apart $(0.5 \mathrm{ml}$ for children over 4 years and $0.25 \mathrm{ml}$ for children $\leqslant 4$ years). In 1995 the influenza vaccine strains were $\mathrm{A} / \mathrm{Taiwan} / 1 /$ 86, A/Johannesburg/34/94, and B/Beijing/ $184 / 93$ according to the recommendations of the World Health Organisation. In 1996, A/Wuhan/359/95 replaced the previous H3N2 component.

Sera were analysed in the Enteric, Respiratory, and Neurological Virus Laboratory, Central Public Health Laboratory, Colindale. Antibodies to the prevalent $\mathrm{A}$ and $\mathrm{B}$ strains were tested by haemagglutination inhibition (HI). Responses were assessed in terms of geometric mean titre (GMT) and protective response. Antibody levels were expressed on a doubling scale $(10,20,40,80$, etc) with a level of 40 or more considered as protective.

\section{STATISTICS}

McNemar's test of paired proportions was used to assess increase in proportion of children exhibiting a protective response. For each strain the child was placed into one of three categories: immune prior to vaccination; protective response made to vaccine; and susceptible after vaccination. Associations with the response made to the vaccine were assessed using the Kruskal-Wallis test and Fisher's exact test for continuous and categorical variables respectively.

A within subject linear model was used to assess increase in antibody titre. The dependent variable in this analysis was the HI titre, which was $\log _{2}$ transformed to remove skewness in this variable. For sera where the antibody level was below the lower limit of detection $(\mathrm{HI}=10)$ a value of 5 was used in this analysis. A blocking factor was fitted for subject. The pre/post-vaccination factor and the interaction between this factor and the variables that may influence response were fitted as within subject effects. The assumption of normally distributed residuals and equality of residual variance were assessed using the Shapiro-Francia 'W' test and the CookWeisburg test respectively.
Table 1 Diagnoses of children in study

\begin{tabular}{lccc}
\hline Diagnosis & $\begin{array}{l}\text { Immunised } \\
\text { children }\end{array}$ & $\begin{array}{l}\text { Paired sera, } \\
\text { non-immunised }\end{array}$ & $\begin{array}{l}\text { Single serum, } \\
\text { non-immunised }\end{array}$ \\
\hline On treatment & & & \\
$\quad$ ALL & 33 & 6 & 1 \\
$\quad$ Relapsed ALL & 1 & 0 & 0 \\
$\quad$ AML & 0 & 2 & 3 \\
$\quad$ Solid tumours & 1 & 10 & 8 \\
Off treatment & & & \\
$\quad$ ALL & 4 & 1 & 1 \\
$\quad$ AML & 1 & 1 & 1 \\
$\quad$ Solid tumours & 2 & 2 & 0 \\
$\quad$ BMT recipients & 0 & $5^{\star}$ & 15 \\
Total & 42 & 27 & \\
\hline
\end{tabular}

ALL, acute lymphoblastic leukaemia; AML, acute myeloid leukaemia; BMT, bone marrow transplant.

*All allogeneic BMT for acute or chronic leukaemia.

\section{Results}

Sera were taken from 67 children in 1995. Of these children, 25 fulfilled the criteria for vaccination and received influenza vaccine. Paired sera were obtained in 27/42 children who were not eligible for immunisation. The remaining 15 children had no follow up serum taken. Twelve of these children died from progressive disease, one was followed up elsewhere, and in two a follow up sample was never taken. A further 17 children were immunised in 1996. Table 1 shows diagnoses of children in the study.

SUSCEPTIBILITY ANALYSIS

Among 84 children tested, 60 (71\%) were susceptible to $\mathrm{H} 1 \mathrm{~N} 1,47(56 \%)$ to $\mathrm{H} 3 \mathrm{~N} 2$, and 58 (69\%) to B. Although the greatest pre-existing protection was to $\mathrm{H} 3 \mathrm{~N} 2$, half of all patients tested were susceptible to this virus. Twenty eight patients $(33 \%)$ were susceptible to all three viruses and only seven patients (8\%) showed protective titres against all three viruses prior to immunisation (data not shown).

Among the 27 unimmunised patients in whom paired sera were available, eight of 26 $(30 \%)$ patients susceptible to at least one virus showed evidence of exposure to influenza A over the winter months (four to $\mathrm{H} 1 \mathrm{~N} 1$, four to H3N2), developing antibody where none was detected previously. Only one of these patients had a blood product transfusion in the three months before the second serum sample, indicating that seroconversion was the result of natural infection.

Ten of 19 (53\%) unimmunised patients with pre-existing full or partial protection lost protective antibody levels to one or more virus types over the winter months. Five patients had blood or platelet transfusions in the two weeks before the initial blood test and may have had false positive tests on the initial screening. However, the other five patients had no history of recent blood product transfusion.

RESPONSE TO IMMUNISATION

Forty two patients were immunised with influenza vaccine. No adverse events were reported. Prior to vaccination, only four patients $(5 \%)$ were fully protected against all three viruses (all had been immunised in 1996 without knowing status), whereas post-vaccination 23 patients $(55 \%)$ were fully protected. Partial protective responses to immunisation were seen in a 
Table 2 Numbers of children making response to each virus subunit

\begin{tabular}{lllll}
\hline \multicolumn{5}{c}{ Pre-vaccination } \\
\cline { 3 - 5 } & & Post-vaccination & \\
\cline { 3 - 5 } & & $H I<40$ & $H I \geqslant 40$ & Total \\
\hline H1N1 & HI $<40$ & 15 & 14 & 29 \\
& HI $\geqslant 40$ & 0 & 13 & 13 \\
& Total & 15 & 27 & 42 \\
H3N2 & HI $<40$ & 7 & 16 & 23 \\
& HI $\geqslant 40$ & 0 & 19 & 19 \\
B & Total & 7 & 35 & 42 \\
& HI $<40$ & 10 & 18 & 28 \\
& HI $\geqslant 40$ & 0 & 14 & 14 \\
& Total & 10 & 32 & 42 \\
\hline
\end{tabular}

Table 3 Response to immunisation by geometric mean titres

\begin{tabular}{llll}
\hline & H1N1 & H3N2 & $B$ \\
\hline $\begin{array}{llll}\text { Pre-vaccination } \\
\text { Geometric mean }\end{array}$ & 12.6 & 23.2 & 12.0 \\
$\begin{array}{c}\text { 95\% CI } \\
\text { Post-vaccination }\end{array}$ & 8.6 to 19.2 & 13.2 to 40.8 & 8.6 to 16.7 \\
$\begin{array}{l}\text { Geometric mean } \\
\text { 95\% CI }\end{array}$ & 60.4 & 124.9 & 48.0 \\
p value & 32.4 to 112.8 & 72.0 to 216.0 & 30.0 to 76.7 \\
& $<0.0001$ & $<0.0001$ & $<0.0001$ \\
\hline
\end{tabular}

further six patients (data not shown). Thus $25 / 38$ patients $(66 \%)$ susceptible to at least one virus made some protective response to the vaccine.

Table 2 shows the numbers of children responding to each virus subunit. The estimated increases in percentage protected against each virus type post-vaccination were: $33 \%$ for $\mathrm{H} 1 \mathrm{~N} 1$ (95\% confidence interval (CI) $17 \%$ to $50 \%, \mathrm{p}=0.0002) ; 38 \%$ for $\mathrm{H} 3 \mathrm{~N} 2$ (95\% CI $21 \%$ to $55 \%, \mathrm{p}=0.0001) ; 43 \%$ for $\mathrm{B}$ (95\% CI $26 \%$ to $60 \%, \mathrm{p}<0.0001)$. Analysis of changes in GMT to each virus following immunisation confirmed a significant response to each virus subunit (table 3 ).

The data were analysed further for factors that might affect response to immunisation. The median age at vaccination of responders was significantly higher than that of nonresponders for $\mathrm{H} 1 \mathrm{~N} 1$ and $\mathrm{H} 3 \mathrm{~N} 2$ (7 years $v 3$ years, $p=0.03$ for both groups) but there was no effect of age on response to B (median age 3 years for responders and non-responders, $\mathrm{p}=0.4)$. There was no correlation between age and the number of protective responses $(0,1$, 2 , or 3$)$ made to the vaccine $(\mathrm{p}=0.9)$. Whereas mean HI titres prevaccination were independent of age, older children showed higher mean post-vaccination titres to $\mathrm{H} 1 \mathrm{~N} 1$ and $\mathrm{H} 3 \mathrm{~N} 2$ but not to B (not shown).

Median total white blood cell count and neutrophil count at the time of immunisation were not significantly different in nonresponders and responders to each virus strain and did not correlate with the number of protective responses obtained (data not shown). However, for the $\mathrm{H} 3 \mathrm{~N} 2$ strain only children with solid tumours produced a higher postvaccination mean $\mathrm{HI}$ titre than those with leukaemia (40.3 24.6 fold increase), and those off treatment produced a better response than those on treatment (19.5 $v 4.2$ fold increase). In neither case was the difference significant $(p=0.06)$, but the numbers of patients in the solid tumour and off-treatment groups were small.

\section{Discussion}

This study confirms previous reports that children with cancer are highly susceptible to influenza virus. Our estimated natural infection rate of $30 \%$ in unimmunised children is similar to that of Borella and Webster, ${ }^{13}$ who reported that $46 \%$ of unimmunised children with leukaemia suffered with a flu like illness in one influenza season. The loss of protective antibody levels in some unimmunised patients noted in this study, possibly as the result of immunosuppressive therapy, might contribute to the increased the risk of clinical infection in children with cancer noted in previous studies. $^{713}$

Previous studies of influenza vaccination in children with cancer have used a variety of different vaccines and have involved both children on treatment for cancer and children who have completed their treatment. ${ }^{13-22}$ All these studies have shown the safety of the vaccine in this patient group. However, the immunogenicity of influenza vaccine in children on chemotherapy varies in different reports according to the type of vaccine used and the viral strains involved. It is clear that two vaccine doses produce a better response than a single dose, ${ }^{14} 15$ and prior exposure to the circulating viral strains seems to increase the likelihood of response to the vaccine. ${ }^{14}{ }^{16}$ Protective antibody levels to individual viral strains following immunisation are reported in $29-75 \%$ of children on chemotherapy, ${ }^{14-16}$ compared to around $70-$ $90 \%$ of healthy children. ${ }^{23}{ }^{24}$ The results of this study are in keeping with an impaired response to vaccination in children with cancer, but nevertheless show a very useful response to immunisation. Similar impaired but useful response to influenza vaccine has been seen in some groups of adult patients receiving chemotherapy. ${ }^{122526}$

By contrast with patients on treatment for cancer, children who have completed their treatment seem to show response rates more comparable with those expected in healthy controls. ${ }^{16-21}$ For H3N2, the response was better in patients off treatment, although the difference was not statistically significant in the small number of patients off treatment in this study. The situation is different in bone marrow transplant recipients, a particularly high risk group with prolonged immunological impairment, who show no response at all until at least six months post-transplant. ${ }^{27}$

In our subgroup analysis we found no significant effect of tumour type (solid tumour versus $\mathrm{ALL}$ ) on response to vaccination, but again a suggestion that response to $\mathrm{H} 3 \mathrm{~N} 2$ was better in solid tumour patients. As all the solid tumour patients were off treatment, the effect of tumour type could not be isolated from on versus off treatment. Children with solid tumours on treatment showed a significantly better response to $\mathrm{A} / \mathrm{New}$ Jersey/8/76 than children being treated for ALL, although no difference was seen for A/Victoria/3/75. ${ }^{16}$ Differences in response between patients with solid and haematological malignancies could 
relate to the intrinsic disease associated immunosuppression of some haematological malignancies as well as the type and intensity of chemotherapy treatment. With the increased intensity of many chemotherapy regimes for solid tumours in recent years, it would be relevant to study response to a trivalent vaccine in a larger number of solid tumour patients currently on treatment.

Our study showed an interesting relation between age and response to immunisation for $\mathrm{H} 1 \mathrm{~N} 1$ and $\mathrm{H} 3 \mathrm{~N} 2$. In the two to three years prior to this study, $\mathrm{H} 3 \mathrm{~N} 2$ was the predominant circulating influenza strain, with limited $\mathrm{H} 1 \mathrm{~N} 1$. Influenza B had been largely absent. ${ }^{28}{ }^{29}$ The effect of age on response to $\mathrm{H} 3 \mathrm{~N} 2$ and $\mathrm{H} 1 \mathrm{~N} 1$ may therefore largely reflect previous exposure or "priming", with older children more likely to have been exposed. Gross found no difference in response to split virus $\mathrm{H} 1 \mathrm{~N} 1$ between 3-5 and 6-18 year olds where H1N1 had been absent from recent natural circulation, ${ }^{23}$ but Wright et al showed a non-significant trend of better response with increasing age for $\mathrm{H} 3 \mathrm{~N} 2$ and $\mathrm{B}$ where these had been the previous circulating strains. ${ }^{24}$ These results support a predominant effect of prior exposure rather than age alone on response.

One remaining area of uncertainty is whether an antibody level of 40, normally considered protective in healthy individuals, is actually protective in the immunocompromised host. In one study, $24 \%$ of unimmunised children with cancer developed proven influenza infection despite influenza virus titres of $\geqslant 1 / 32,{ }^{6}$ but another study reported an incidence of flu like illness in only $10 \%$ of immunised children with leukaemia, compared to $46 \%$ of unimmunised children, suggesting that immunisation was associated with a reduced incidence of clinical infection. ${ }^{13}$ The latter study showed a rise in antibody titres following immunisation, but post-immunisation titres were not discussed in relation to clinical infection in individual patients. The discrepancy between the studies could be explained if there is high rate of loss of protective antibody levels in patients receiving chemotherapy, a finding shown in the current study. If the latter explanation is correct, it may be that annual immunisation prior to the influenza season in children with cancer is of greater benefit than suggested by the change in the proportions of patients with protective antibody titres following immunisation, because it may reduce loss of protective immunity in some patients as well as facilitating new protective immunity in others. In one study of leukaemic children on and off treatment, antibody levels returned to baseline by 12 months following vaccination, ${ }^{17}$ but in other children, mainly off treatment, levels were maintained at least until six months following vaccination, ${ }^{21} 22$ suggesting that protection would be maintained during the highest risk time for influenza even if it is subsequently lost. Taken with previous data, our study suggests that the risk of influenza infection in children with cancer may be reduced substantially by immunisation.
Even though influenza usually runs a mild course in children with cancer, the occasional morbidity and mortality and high risk of interruption of potentially curative chemotherapy make it worth preventing. It seems sensible that the household contacts of such children and the hospital staff caring for them, as well as the children themselves, should receive annual immunisation against influenza, ${ }^{5} 1030$ although to our knowledge there is no direct evidence to support this approach. When proven influenza occurs despite immunisation, judicious use of zanamivir may reduce hospital admission rates and complications in high risk patients, but data in these groups are lacking. ${ }^{31}$

In conclusion, this study supports existing recommendations that all children with cancer receiving chemotherapy should receive annual immunisation with influenza vaccine. We suggest that their household contacts and appropriate hospital staff should also be immunised. Two doses of vaccine should be given at the first immunisation but data are needed on whether one or two doses of vaccine are required for second and subsequent vaccinations in patients on chemotherapy. Further data are also needed on the efficacy of the vaccine in children with solid tumours on treatment.

JCC was supported by a Leukaemia Research Fund Clinical Fellowship. CRP is supported by the Cancer Research Campaign. The authors are grateful to Aventis Pasteur MSD for Campaign. The authors
supply of the vaccine.

1 Yousef HM, Englund J, Couch R, et al. Influenza among hospsitalised adults with leukaemia. Clin Infect Dis 1997;24:1095-9.

2 Elting LS, Whimbey E, Lo W, et al. Epidemiology of influenza A virus infection in patients with acute or chronic leukaemia. Support Care Cancer 1995;3:198-202.

3 Whimbey E, Elting LS, Couch RB, et al. Influenza A virus infections among hospitalized adult bone marrow transplant patients. Bone Marrow Transplant 1994;13:437-40

4 Whimbey E, Champlin RE, Couch RB, et al. Community respiratory virus infections among hospitalised adult bone marrow transplant recipients. Clin Infect Dis 1996;22:77882 .

5 Hall CB. Influenza - a shot or not? Pediatrics 1987;79: 564-5.

6 Kempe A, Hall CB, MacDonald NE, et al. Influenza in chilKempe A, Hall CB, MacDonald NE, et al.
dren with cancer. P Pediatr 1989;115:33-9.

7 Feldman S, Webster, RG, Sugg M. Influenza in children and young adults with cancer. Cancer 1977;39:350-3.

8 Potter MN, Foot, ABM, Oakhill. Influenza A and the virus associated haemophagocytic syndrome: cluster of three cases in children with acute leukaemia. F Clin Pathol 1991; 44:297-9.

9 Department of Health. Immunisation against infectious disease. London: HMSO, 1996:113-20.

10 Advisory Committee on Immunisation Practices (ACIP) of the US Public Health Service. Prevention and control of influenza. MMWR 1999;48:1-23.

11 Irish C, Alli M, Gilham C, et al. Influenza vaccine uptake and distribution in England and Wales, July 1989-June 1997. Health Trends 1998;30:51-5.

12 Gross PA, Gould L, Brown AE. Effect of cancer chemotherapy on the immune response to influenza virus vaccine: review of published studies. Rev Infect Dis 1985;7:613-18.

13 Borella L, Webster RG. The immunosuppressive effects of long term combination chemotherapy in children with acute lymphoblastic leukemia in remission. Cancer Res 1971;31:420-6.

14 Allison JE, Glezen P, Taber LH, et al. Reactogenicity and immunogenicity of bivalent influenza $\mathrm{A}$ and monovalent influenza B virus vaccines in high-risk children. F Infect Dis 1997;136(suppl):S672-6.

15 Sumaya CV, Williams TE, Brunell PA. Bivalent influenza vaccine in children with cancer. F Infect Dis 1997; 136(suppl):S656-60.

16 Gross PA, Lee H, Wolff JA, et al. Influenza immunization in children with cancer. F Pediatr 1978;92:30-5.

17 Smithson WA, Siem RA, Ritts RE, et al. Response to influenza virus immunisation in children receiving chemotherapy for malignancy. $\mathcal{F}$ Pediatr 1978;93:632-4

18 Lange B, Shapiro SA, Waldman MTG, et al. Antibody responses to influenza immunization of children with acute lymphoblastic leukemia. F Infect Dis 1979;3:402-6. 
19 Steinherz PG, Brown AE, Gross PA, et al. Influenza immunization of children with neoplastic diseases. Cancer 1980 45:750-6.

20 Brown AE, Steinherz PG, Miller DR, et al. Immunization against influenza virus in children with cancer: results of a three-dose trial. F Infect Dis 1982;145:126.

21 Brydak LB, Rokicka-Milewska R, Jackowska T, et al. Kinetics of humoral response in children with acute lymphoblastic leukemia immunized with influenza vaccine in 1993 in Poland. Leuk Lymphoma 1997;26:163-9.

22 Brydak LB, Rokicka-Milewska R, Machala M, et al. Immunogenicity of subunit trivalent influenza vaccine in children with acute lymphoblastic leukemia. Pediatr Infect Dis 1998; 17:125-9.

23 Gross PA. Reactogenicity and immunogenicity of bivalent influenza vaccine in one and two-dose trials in children: a summary. F Infect Dis 1997;136(suppl):S616-25.

24 Wright PF, Cherry JD, Foy HM, et al. Antigenicity and reactogenicity of influenza A/USSR/77 virus vaccine in children - a multicentred evaluation of dosage and safety. Rev Infect Dis 1983;5:758-64.
25 Lo W, Whimbey E, Elting L, et al. Antibody response to a two-dose influenza vaccine regimen in adult lymphoma atients on chemotherapy. Eur 7 Clin Microbiol Infect Dis 993;12:778-82.

26 Anderson H, Petrie K, Berrisford C, et al. Seroconversion after influenza vaccination in patients with lung cancer. $\mathrm{Br}$ f Cancer 1999;80:219-20.

27 Engelhard D, Nagler A, Hardan I, et al. Antibody response to a two-dose regimen of influenza vaccine in allogeneic $\mathrm{T}$ cell-depleted and autologous BMT recipients. Bone Marrow Transplant 1993;11:1-5.

28 Hutchinson EJ, Joseph CA, Zambon MC, et al. Influenza surveillance in England and Wales. Oct 1995-June 1996. Commun Dis Respir Rev 1996;6:R163-9.

29 Dedman D, Joseph CJ, Zambon MC, et al. Influenza surveillance in England and Wales. Oct 1996-June 1997. Commun Dis Respir Rev 1997;7:R212-19.

30 Isaacs D. Influenza immunization: time to stop the charade. Curr Opin Pediatr 1995;7:3-5.

31 Nguyen-Van-Tam JS. Zanamivir for influenza: a public health perspective. BMF 1999;319:655-6.

\section{Immunisation debates}

Whether we like it or not "the media" are the most powerful influence on public opinion. There have been times when unbalanced reporting has done harm but recently (December 2000-January 2001) there has been evidence of a well-considered approach, at least to the subject of immunisation. The apparent success of group $\mathrm{C}$ meningococcal vaccination has been well publicised and MMR vaccination defended. Commenting on a recent fall in MMR acceptance rates, the Times concluded (January 5, 2001) that parents who refuse the vaccine for their children "shirk, for selfish reasons and on the basis of wholly inconclusive scientific research, a manifest social responsibility". Strong stuff, and probably unfair to many concerned parents, but at least fighting on the side of the angels.

Whenever questions are raised about immunisation there are fears about vaccine refusal, the great spectre being the example of pertussis in the early 1980s when disease rates soared after fears of vaccine-induced encephalopathy resulted in low acceptance rates. But questions must be asked. It may be irresponsible to endanger vaccination programmes on the basis of inadequate data but it is never irresponsible to ask sensible questions.

There is no doubt that parents do have their own thoughts about immunisation. In a national survey in the USA (Bruce G Gellin and colleagues. Pediatrics 2000;106:1097-102) 23\% agreed that "children get more immunisations than are good for them", and $25 \%$ agreed, or expressed concern, that "too many immunisations could weaken the child's immune system". Could such concerns have any rational basis? Immunisation almost certainly has non-specific effects on immune function. Both measles and BCG immunisations probably boost immunity in non-specific as well as specific ways and so may other immunisations. In a randomised study in Germany (S Otto and colleagues. Fournal of Infection 2000;41:172-5) babies who had their first immunisations at 3 months had more symptoms (cough, snuffles, restlessness, rash, or apparent pain) during their third month than did babies immunised at 2 months. The effects may be different in countries with high infection rates and high mortality. In Guinea-Bissau, West Africa (Ines Kristensen and colleagues. BMF 2000;321:1435-8) measles and BCG immunisation improved non-specific immunity in young children but diphtheria, pertussis, and tetanus (DPT), and poliomyelitis immunisation appeared to impair it. This effect may reduce the effectiveness of DPT and polio immunisation and in this study DPT immunisation was associated with an increase in early childhood mortality. The BMF published the paper together with a commentary which questioned the reliability of the results and an editorial which strongly defended current immunisation practice and hinted that there were those who thought that the paper should not have been published.

The television science guru Jacob Bronowski once wrote, "ask an impertinent question, and you are on the way to a pertinent answer". No subject, not even immunisation, is sacrosanct. Scaremongering is silly and irresponsible but questions have to be asked and debated. Evidence resolved the pertussis controversy and evidence has assuaged MMR fears. Parents' concerns must be faced and answered. Recent evidence suggests that the media can be an ally rather than an irritant in this process.

ARCHIVIST 